Original Article

\title{
Single Burrhole Craniostomy with Subdural Placement of Foley Catheter for Drainage of Chronic Subdural Hematoma
}

\author{
Faiq Sheikh ${ }^{1}$, Jamal Nasir², Adnan Khalid', Rabia Saleem ${ }^{1}$, Fakiha Sheikh ${ }^{1}$, Abuzar Rauf ${ }^{1}$ \\ ${ }^{1}$ Department of Neurosurgery, Punjab Institute of Neurosciences (PINS), Lahore, Pakistan \\ ${ }^{2}$ Children Hospital and the Institute of Child Health, Lahore - Pakistan
}

\section{ABSTRACT}

Objective: Chronic subdural hematoma (CSDH) is a common neurosurgical condition of the elderly. The lack of consensus over a single standard surgical procedure for the management of CSDH urges neurosurgeons to evaluate different techniques in terms of a better outcome.

Material \& Methods: In this prospective study forty cases of CSDH who had undergone single burr-hole craniostomy and subdural placement of Foley catheter were analyzed. The observations were made concerning clinical, radiological findings, postoperative complications, and mortality rate.

Results: The study included 26 males and 14 females with a mean age of 67 years. The most common presenting symptom was hemiparesis $(n=18,45 \%)$. Recurrent hematoma and recurrent evacuation rate was $10 \%$. There was no case of newly developed postoperative seizures, pneumocephalus, intracranial hemorrhage, and wound infection. The mortality rate during the study was $0.0 \%$. The mean Glasgow outcome score (GOS) at the time of discharge was 4.8.

Conclusion: We concluded that single burr-hole evacuation of CSDH with Foley catheter placement appears to be an effective procedure with a significant reduction in postoperative pneumocephalus. Factors such as sudden decompression of hematoma, the air in the subdural cavity, misplacement of drainage tubes, and damage to blood vessels during surgery can be reduced with this technique.

Keywords: Chronic subdural hematoma (CSDH), Pneumocephalus, Intracranial Hemorrhage (ICH).

Abbreviations: CSDH: Chronic subdural hematoma. ICH: Pneumocephalus, Intracranial Hemorrhage.

Corresponding Author: Abuzar Rauf

Department of Neurosurgery

Punjab Institute of Neurosciences (PINS), Lahore - Pakistan

Email: abuzar9507@gmail.com

Date of Submission: 11-05-2021

Date of Revision: 28-06-2021

Date of Online Publishing: 30-06-2021

Date of Print: 30-06-2021

DOI: $10.36552 /$ pjns.v25i2.567

\section{INTRODUCTION}

Chronic subdural hematoma (CSDH) is one of the most frequent neurosurgical diseases in patients $>70$ years of age with an incidence of 3.4-5 per 100,000 people per year. ${ }^{1}$ Exact etiology and pathophysiology are not completely understood. According to traditional theory, it isa slowly growing encapsulated collection of fluid, blood, 
and its products between the dura and arachnoid matter formed due to tearing of bridging veins following minor head trauma. ${ }^{3}$ The outer membrane of this encapsulated CSDH has highly permeable microcapillaries and gap junctions that allow blood to leak in the cavity lead to the enlargement of hematoma. It has been recently suggested that the development and propagation of CSDH involves a complex pathway of inflammation, inflammatory cytokines, angiogenesis, and fibrinolysis. ${ }^{4}$

As the disease progress blood and its breakdown products reabsorbed over time. However, patients become symptomatic due to brain compression caused by the hematoma or due to brain irritation from blood breakdown products. The clinical presentation includes altered states of consciousness, focal neurological deficit, headache, seizures, speech abnormalities, and cognitive dysfunction. The CSDH is usually diagnosed by computed tomography (CT) brain appearing as a hypodense, isodense, or mix density crescent lesion adjacent to the inner table. Conservative and non-conservative methods are used to treat patients with $\mathrm{CSDH}$. Surgical evacuation of hematoma is the treatment of choice for neurologically symptomatic patients and those who have failed non-surgical treatment. Three commonly done procedures are twist-drill craniostomy, burr-hole craniostomy, and craniotomy. ${ }^{5-6}$ These techniques are often supplemented with other procedures such as intraoperative irrigation and postoperative drainage. Burr-hole craniostomy with irrigation and placement of closed drainage system is the most commonly practiced surgical technique so far. Twist drill craniostomy is associated with higher recurrence rates as compared to burr-hole technique and postoperative morbidity is high with craniotomy technique. ${ }^{6-7}$

One of the biggest challenges in developing countries is working with limited resources in terms of the availability of trained medical staff and equipment. The simplicity and cost- effectiveness play a major role in the selection of surgical procedures, particularly in rural districts. We aimed to discover a relatively safe, effective, minimally invasive, economical surgical technique suitable for everyday practice. This study is performed to evaluate the outcome of singleburr-hole drainage of $\mathrm{CSDH}$ with Foley catheter placement in subdural space without prior irrigation. We prefer the use of a Foley catheter as it is a self-retainable, less traumatic, low-cost catheter as compared to standard drainage systems.

\section{MATERIALS AND METHODS}

\section{Study Setting}

This study was conducted at the Neurosurgery Department of Punjab Institute of Neurosciences (PINS) after approval from the ethical review board of the institute. Total 40 patients of CSDH underwent single burr-hole craniostomy and they were reviewed prospectively from March 2019 to December 2019. Prior ethical approval was taken from the institution's IRB committee. Patients data was collected with informed consent.

\section{Inclusion Criteria}

Patients of either gender with more than 18 years of age with radiographic findings of subdural hematoma of greater than three weeks were included in the study.

\section{Exclusion Criteria}

Patients with vascular malformations, subdural empyema, subdural hygroma, multiple loculated, organized or calcified, and recurrent chronic subdural hematomas were excluded.

\section{Surgical Procedure}

Patients fulfilling inclusion criteria were included in the study and informed consent was taken. These patients were further assessed through a 
detailed history, including biodata, onset, and progression of symptoms, history of fall or minor head trauma, chronic medical conditions, medications, and alcohol or drug abuse. A thorough physical and neurological examination was performed followed by necessary investigations. The confirmation of CSDH was done by non-contrast computed tomography (CT) and radiographic features such as location and maximum thickness of the hematoma, presence of midline shift (MLS) of more than $5 \mathrm{~mm}$, mass effect determined by the effacement of the sulci or compression of ventricles, were noted. The procedure was done under general anesthesia and aseptic measures. Patients were kept in the supine position and an incision site was marked at the dependent area (maximum thickness on CT brain). After making a linear incision of around $3 \mathrm{~cm}$, a single burr-hole craniectomy was done and the dura was exposed. Durotomy was done in a punctate manner and membrane penetration was confirmed as cavity fluid oozed out. Foley catheter of $10 \mathrm{fr}$ was placed inside the subdural space and balloon was instilled with saline $(3 c c)$, and then the catheter was pulled back until stuck. A drainage bag was attached (non-suction type) and the drain was kept in place for at least 3 days.

\section{RESULTS}

\section{Gender \& Age Distribution}

Forty patients underwent single burr-hole craniostomy with 26 males and 14 females. The mean age was 66.95 years.

\section{Preoperative GCS}

On admission six cases had GCS 15, 14 cases had GCS $13-14,16$ cases had GCS $9-12$ and 4 patients had GCS less than 8.

\section{Presenting Features}

The presenting clinical features include hemiparesis ( $n=18,45 \%)$, hemiplegia $(n=2,5 \%)$, sphincter incontinence $(n=1,2.5 \%)$, seizures $(n=1,2.5 \%)$, headache $(n=6,15 \%)$, aphasia $(n=$ $6,15 \%)$ and decreased level of consciousness GCS $<13(n=20,50 \%)$. Other medical conditions include moderate to poorly controlled hypertension $(n=10,25 \%)$ and type 2 diabetes $(n=6,15 \%)$. Six patients were taking antiplatelet medications and history of minor head trauma was positive in seven patients.

\section{Indications of Subdural Hematomas}

On non-contrast $\mathrm{CT}$ brain, the right-sided chronic subdural hematoma was present in 9 cases. Leftsided hematoma in 26 and the bilateral hematoma were present in 5 cases. Preoperative estimated mean hematoma volume based on CT findings was $101.8 \mathrm{ml}$. After the surgery within 48-hour, it was $24.6 \mathrm{ml}$.

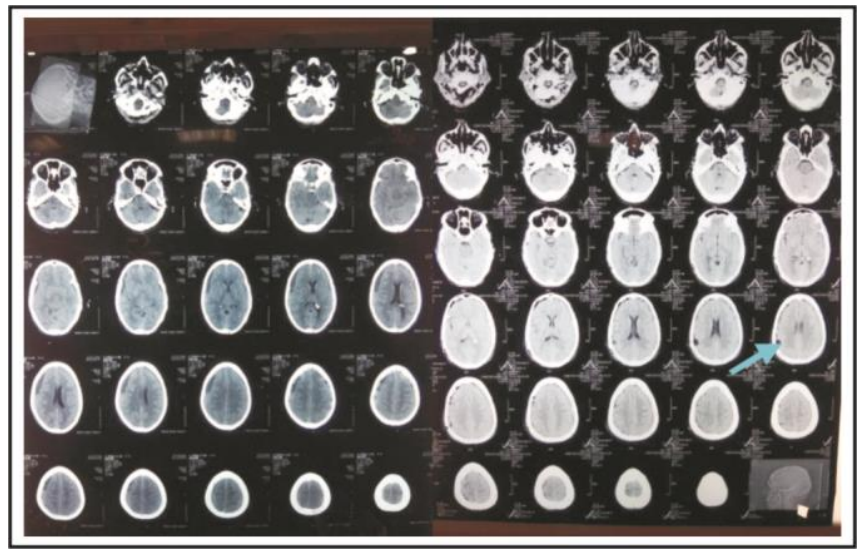

Figure 1: Pre-Op CT Brain showing bilateral Chronic SDH more marked on the right side and Post-Op image showing almost complete resolution of hematoma with an arrowhead pointing to infiltrated balloon of the catheter.

\section{Postoperative GCS}

In the postoperative period, most patients had improved GCS (13 - 15) with only 2 patients (5\%) had GCS 9 - 12. Glasgow outcome score (GOS) at the time of discharge was 5 in 32 patients (80\%), 4 in 7 patients (17.5\%), 3 in 1 patient (2.5\%). See Table 1 for details. 
Table 1: General Findings In Patients.

$\begin{array}{lc}\text { Findings } & \begin{array}{c}\text { No. of } \\ \text { Patients (\%) }\end{array} \\ \text { Gender (Total Patients = 40) } & \\ \text { Male } & 26(65 \%) \\ \text { Female } & 14(35 \%) \\ \text { Mean Age in Years = 67 years } & \\ \text { Clinical Presentation } & \\ \text { GCS < 13 } & 20(50 \%) \\ \text { Hemiparesis } & 18(45 \%) \\ \text { Hemiplegia } & 2(5 \%) \\ \text { Headache } & 6(15 \%) \\ \text { Seizures } & 1(2.5 \%) \\ \text { Aphasia } & 6(15 \%) \\ \text { Sphincter Incontinence } & 1(2.5 \%) \\ \text { Patients on Anti-Platelet Medication } & 6(15 \%) \\ \text { Patients with History of Head Trauma } & 7(17.5 \%) \\ \text { Pre-Operative CT Findings } & \\ \text { Left Sided Subdural Hematoma } & 26(65 \%) \\ \text { Right Sided Subdural Hematoma } & 9(22.5 \%) \\ \text { Bilateral Subdural Hematoma } & 5(12.5 \%) \\ \text { Pre-Operative Mean Hematoma Vol. = } & \mathbf{1 0 1 . 8 ~} \mathbf{m l}\end{array}$

\section{Outcomes}

All patients were examined postoperatively concerning improvement in presenting symptoms and neurological status. CT scan was done $24-48$ hours after the operation to measure a decrease in hematoma thickness and also to exclude pneumocephalus and re-bleed. Recurrence of $\mathrm{CSDH}$ is defined as re-accumulation of hematoma fluid that needs re-operation following primary treatment. Drain efficacy was monitored using both radiographic and clinical markers (volume of fluid in drain bag, findings of postoperative scan, postoperative symptoms, and complications). Functional outcome was assessed by using the Glasgow outcome scale $(1-5)$ at the time of discharge. CT imaging was also performed on clinical follow-up after 3 months.

\section{Cost-effectiveness}

The cost of using Foley's catheter as compared to CDIS (Close drainage and irrigation system) is very low as shown in Table 2.
Table 2: Comparison in terms of cost-effectiveness using Foley's catheter vs. closed drainage and irrigation system (CDIS).

\begin{tabular}{lll} 
& Foley's Catheter & CDIS \\
\multirow{2}{*}{ Cost per unit } & PKR400 & PKR30000 \\
Total estimated & USD 2.54 & USR 16000 190.17 \\
cost & USD 101.43 & PKR1200000 \\
\hline
\end{tabular}

\section{Complications}

Four (10\%) patients developed a recurrent hematoma. All cases of recurrence were further managed with surgical evacuation (re-exploration of same burr-hole). There was no case of postoperative pneumocephalus, intracranial hemorrhage, seizures, and surgical site infection, or subdural empyema. The mortality rate during the study was $0.0 \%$. No patient required craniotomy post-operatively. One patient suffered from a respiratory tract infection treated with antibiotics. See Table 3 for details.

Table 3: Post-Operative Findings.

\begin{tabular}{|c|c|}
\hline Findings & $\begin{array}{l}\text { No. of Patients } \\
\text { (\%) }\end{array}$ \\
\hline \multicolumn{2}{|l|}{ Clinical Findings } \\
\hline GCS $13-15$ & $38(95 \%)$ \\
\hline GCS 9-12 & $2(5 \%)$ \\
\hline \multicolumn{2}{|c|}{ Post-Operative Mean Hematoma Vol. = $24.6 \mathrm{ml}$} \\
\hline Recurrence of Hematoma & $4(10 \%)$ \\
\hline Need For Craniotomy & $0(0.0 \%)$ \\
\hline \multicolumn{2}{|l|}{ Post-Operative Complications } \\
\hline Intracranial Hematoma/ Bleed & $0(0.0 \%)$ \\
\hline Post-Operative Seizures & $0(0.0 \%)$ \\
\hline Infection (Wound, Subdural Empyema) & $0(0.0 \%)$ \\
\hline Pneumocephalus & $0(0.0 \%)$ \\
\hline Resp. Tract Infection & $1(2.5 \%)$ \\
\hline Post-Operative Mortality & $0(0.0 \%)$ \\
\hline \multicolumn{2}{|c|}{$\begin{array}{l}\text { Mean Glasgow Outcome Score at the Time of } \\
\text { Discharge }=4.8\end{array}$} \\
\hline
\end{tabular}

\section{DISCUSSION}

Chronic subdural hematoma is a common neurosurgical condition that is considered to be an important reversible cause of disability in the 
geriatric population. The treatment is often surgical evacuation, using techniques such as twist drill craniostomy, burr-hole craniostomy, and craniotomy. Many studies have been conducted to rule out the most effective and safe scheme among these procedures but no conclusion has yet been reached. ${ }^{6,7}$ Burr-hole craniostomy is the most widely practiced procedure with differences in operative technique like the number of holes, intraoperative irrigation or no irrigation, and postoperative drainage with the type of drain systems. Although burr-hole craniostomy with irrigation and subdural drainage is the most effective method for evacuation of $\mathrm{CSDH}$, its overall performance in terms of a good outcome is still controversial. Good outcome results from the better recovery of patients, low perioperative mortality, and low postoperative complications. In literature, these complications include systemic medical ailments, recurrence of hematoma, infection (wound or intracranial), postoperative seizures, pneumocephalus, and intracranial hemorrhage following surgical procedure. $1,5,8,9,10$

Rhode et al performed a retrospective study to review the complications following burr-hole craniostomy and close system drainage $(B C D)$ for CSDH evacuation ${ }^{8}$ Seventy-seven (20.5\%) patients encountered surgical complications with most frequent minor complication postoperative seizure occurred in fifty-one patients (13.5\%) and major complication intracranial hemorrhage occurred in 13 patients (3.4\%). Out of 13, 8 cases (2.1\%) were intracerebral hemorrhage and 5 (1.3\%) were epidural hematoma. Subdural empyema was reported in 8 cases $(2.1 \%)$. The surgical mortality rate was $1.1 \%$ with an overall mortality of $13.3 \%$. The rate of medical complications was $15.7 \%$ with pulmonary complications being most frequent $7.7 \%$. A similar type of study was conducted by Lee et al to review complications after $B C D$ for subdural lesions. ${ }^{9}$ They noticed that postoperative acute intracranial hemorrhage was developed in 14
(3.5\%) patients with epidural hematoma in 7 (1.7\%) and acute SDH in 6 (1.5\%) cases. Postoperative seizures were noted in 8 (2.0\%) patients. Surgical or management errors occur in $7(1.7 \%)$ cases including 4 cases of malpositioning of a drainage catheter in brain parenchyma. Eighty-eight (22.3\%) patients suffered nonsurgical complications with $7.4 \%$ pulmonary problems. Zumofen et al. reported a hematoma persistence or recurrence rate of $13.1 \%$ in a study for $\mathrm{CSDH}$ evacuation with burr-hole trepanation and subperiosteal drain. ${ }^{5}$ Postoperative seizure rate within 72 hours of surgery was $6.6 \%$ and the infection rate was $1.6 \%$. The overall complication rate was $10.9 \%$ with re-intervention of surgery in $9.3 \%$ of cases. The perioperative mortality rate was $3.4 \%$ with 3 patients who expired from direct surgical complications (two patients continued to bleed from the subdural membrane and one patient from immediate postoperative epidural bleed). Chen et al. observed a recurrence rate of $13.4 \%$ after single burr-hole drainage of CSDH. Postoperative complications occurred in $27.3 \%$ of patients with $5.1 \%$ cases of acute intracranial hemorrhage. ${ }^{1} 100$ patients of $\mathrm{CSDH}$, who had undergone surgical management mostly by burrhole craniostomy and drainage were reviewed by menses et al. Residual hematoma was noted in 18 (18\%) cases with each case underwent a new surgical procedure. Postoperative pneumocephalus was found in 11 (11\%) cases. The mortality rate was $2 \%{ }^{10}$

Two well-mentioned complications after surgical evacuation of $\mathrm{CSDH}$, are recurrence of hematoma fluid with overall rate ranges from $0.36 \%$ to $33.3 \%{ }^{1,15,16,23}$ and postoperative pneumocephalus has seen in $11 \%$ of cases. ${ }^{10,17}$ The commonly proposed causes for recurrence are poor re-expansion of the brain, presence of intracranial air, fragile cortical vessel injury, and rise in cerebral blood flow following the evacuation of hematoma. ${ }^{18}$ Burr-hole craniostomy with drainage and irrigation sharply reduces intracranial pressure causing damage to 
traversing vessels that leads to re-accumulation of blood. This abrupt decline in pressure also helps influx of air in subdural space that may lead to the development of tension pneumocephalus (accumulated air increases intracranial pressure causing brain herniation and neurological deterioration). ${ }^{17}$ Long-standing compressed brain and use of suction-type drainage systems also contribute to the entrance of intracranial air. This air in the subdural cavity prolongs the widening of the subdural space, blocks the re-expansion of the brain following the evacuation, increases recurrence rate, and prolongs recovery time. ${ }^{19,23}$ Reducing the chances of subdural air during surgery is of high importance for a better outcome of any procedure. The recurrence rate in our study was $10 \%(n=4)$ and no case of postoperative pneumocephalus was observed. All patients of recurrence were further managed with re-exploration of the same burr-hole. No patient required an additional surgical procedure.

An intracranial hemorrhage following $\mathrm{CSDH}$ evacuation is a rarely noticed major complication (incidence $\sim 0.7-5 \%)^{1,8,9,12}$ In literature, there are case reports of the development of remote (contralateral) hemorrhage, acute subdural, epidural, intracerebral bleed, and intraparenchymal hemorrhage after surgical evacuation of $\mathrm{CSDH}^{11-15}$ Although exact pathophysiology is unknown sudden changes in cortical blood flow following rapid decompression of long-standing $\mathrm{CSDH}$, defective vascular auto-regulation and traumatic injury to cerebral vasculature secondary to drain placement may lead to this condition. ${ }^{13-14}$ Presenting technique in our study allows slow spontaneous drainage of hematoma and causes less direct trauma to blood vessels and brain tissue. No cases of traumatic handling, malpositioning of the drain, and intracranial bleed were reported in this study.

Surgical site infection involves superficial skin infection and deep subdural infection or empyema. The rich blood supply of the $\mathrm{CSDH}$ membrane makes it a potential site for infections with drainage of CSDH being the most common neurosurgical procedure associated with subdural empyema. The reported cases of subdural empyema that had undergone burr-hole evacuation with or without subdural drain are $2 \% .^{8,22}$ These infections further add strain to case management as treatment involves a high dose of antibiotics and surgical drainage of pus. No case of surgical site infections was noted in this study. Postoperative seizures incidence is $2-13 \% \%^{5,8,9,21}$ after surgical evacuation of $\mathrm{CSDH}$. Chen et al noted that the incidence of early postoperative seizures (within 3 weeks of surgery) was 5.4\% after burr-hole drainage of $\mathrm{CSDH}^{21}$ In our study, no case of postoperative seizure was observed. Postoperative morbidities affect the overall cost of treatment in terms of longer hospital stays and the need for re-interventions. The low rate of complications and selection of low-cost drainage apparatus make this procedure cost-efficient.

The result of this study shows that single burrhole evacuation of CSDH via subdural placement of Foley catheter without prior irrigation seems to be an effective procedure. This simple technique helps to reduce factors such as sudden decompression of hematoma, acute drop in intracranial pressure, brain re-expansion failure due to air in subdural space, tearing of bridging vessels, and drainage tube related injuries. It allows minimally invasive, slow, spontaneous, drainage of hematoma with the help of a less traumatic non-suction type of Foley catheter. Reduction in postoperative pneumocephalus, an important factor for recurrence makes this procedure a good choice for the treatment of chronic subdural hematoma, although large series of studies are needed to compare its long-term performance with other procedures.

\section{REFERENCES}

1. Chen FM, Wang K, Xu KL, et al. Predictors of acute intracranial hemorrhage and recurrence of chronic subdural hematoma following burr-hole drainage. 
BMC Neurol. 2020; 20 (1): 92.

2. Hwang SC, Im SB, Kim BT, Shin WH. Safe entry point for twist-drill craniostomy of a chronic subdural hematoma. Journal of Neurosurgery, 2009; 110 (6): 1265-70.

3. Markwalde TM. Chronic subdural hematoma: a review. J Neurosurgery, 1981; 54 (5): 637-45.

4. Edlmann E, Giorgi-Coll S, Whitfield PC, Carpenter $\mathrm{KLH}$, Hutchinson PJ. Pathophysiology of chronic subdural hematoma: inflammation, angiogenesis and implications for pharmacotherapy J Neuroinflammation, 2017; 14: 108.

5. Zumofen $D$, Regli L, Levivier $M$, Krayenbühl N. Chronic subdural hematomas treated by burr-hole trepanation and a subperiostal drainage system. Neurosurgery, 2009; 64 (6): 1116-22.

6. Weigel R, Schmiedek P, Krauss JK: Outcome of contemporary surgery for chronic subdural hematoma: evidence-based review, 2003; 74 (7): 937-43.

7. Santarius T, Kirkpatrick PJ, Kolias AG, Hutchinson PJ. Working toward rational and evidence-based treatment of chronic subdural hematoma. Clin Neurosurg. 2010; 57: 112-22.

8. Rohde V, Graf G, Hassler W. Complications of burrhole craniostomy and closed-system drainage for chronic subdural hematomas: a retrospective analysis of 376 patients. Neurosurg Rev. 2002; 25 (1-2): 89-94.

9. Lee HS, Song SW, Chun YI, Choe WJ, et al. Complications Following Burr-Hole Craniostomy and Closed-System Drainage for Subdural Lesions. Korean J Neurotrauma, 2018; 14 (2): 68-75.

10. Sikahall-Meneses $E$, Salazar-Pérez N, SandovalBonilla B. Chronic subdural hematoma: surgical management in 100 patients. Cirugia Y Cirujanos, 2008; 76 (3): 199-203.

11. Sousa J. Golash A. VazJ, et al. Spontaneous intracerebral hemorrhage following evacuation of chronic subdural hematomas J Clin Neurosci. 2004; 11: 794-796.

12. Muneza S, Rasoloherimampiononiaina MR,
Nduwamariya MJ. Postoperative intracerebral and intraventricular hemorrhages following removal of a chronic subdural hematoma. Journal of Clinical Neuroscience, 2009; 16 (10): 1346-8.

13. Ulivieri $S$, Olivieri $G$. Intracerebral haemorrhage following surgical evacuation of chronic subdural haematoma: Case report. The Journal of Surgery, 2008; 29 (5): 233-4.

14. Cohen-Gadol AA. Remote contralateral intraparenchymal hemorrhage after over drainage of a chronic subdural hematoma, 2013; 4 (10): 834-836.

15. Kim $\mathrm{CH}$, Song GS, Kim $\mathrm{YH}$, et al. Remote Hemorrhage after Burr-Hole Drainage of Chronic Subdural Hematoma, 2017; 13 (2): 144-148.

16. Almenawer SA, Farrokhyar F, Hong C, et al. Chronic subdural hematoma management. Ann Surg. 2014; 259: 449-57.

17. Ihab Z. Pneumocephalus after surgical evacuation of chronic subdural hematoma: Is it a serious complication? Asian J Neurosurg. 2012; 7: 66-74.

18. Chang $\mathrm{SH}$, Yang $\mathrm{S}$, Son $\mathrm{BC}$, et al. Cerebellar hemorrhage after burr-hole drainage of supratentorial chronic subdural hematoma. J Korean Neurosurg Soc. 2009; 46: 592-5.

19. You CG, Zheng XS. Postoperative pneumocephalus increases the recurrence rate of chronic subdural hematoma. Clin Neurol Neurosurg. 2018 Mar; 166: 56-60.

20. Sharma BS, Tewari MK, Khosla VK, et al.Tension pneumocephalus following evacuation of chronic subdural hematoma, 1989; 3 (3): 381-387.

21. Chen CW, Kuo JR, Lin HJ, et al. Early post-operative seizures after burr-hole drainage for chronic subdural hematoma: correlation with brain CT findings. J Clin Neurosci. 2004; 11 (7): 706-9.

22. Pencalet P. Complications of chronic subdural hematoma in the adult, 2001; 47 (5): 491-4.

23. Ohba S, Kinoshita Y, Nakagawa T, Murakami H. The risk factors for recurrence of chronic subdural hematoma. Neurosurg Rev. 2013; 36: 145-9. 


\section{Additional Information}

Disclosures: Authors report no conflict of interest.

Ethical Review Board Approval: The study was conformed to the ethical review board requirements.

Human Subjects: Consent was obtained by all patients/participants in this study.

Conflicts of Interest:

In compliance with the ICMJE uniform disclosure form, all authors declare the following:

Financial Relationships: All authors have declared that they have no financial relationships at present or within the previous three years with any organizations that might have an interest in the submitted work.

Other Relationships: All authors have declared that there are no other relationships or activities that could appear to have influenced the submitted work.

\section{AUTHORS CONTRIBUTIONS}

\begin{tabular}{|l|l|l|}
\hline Sr.\# & Author's Full Name & Intellectual Contribution to Paper in Terms of: \\
\hline 1. & Faiq Sheikh & Study design and methodology. \\
\hline 2. & Fakiha Sheikh & Paper writing, referencing, and data calculations. \\
\hline 3. & Rabia Saleem & Data collection and calculations. \\
\hline 4. & Adnan Khalid & Analysis of data and interpretation of results etc. \\
\hline 5. & Jamal Nasir & Literature review and manuscript writing. \\
\hline 6. & Abuzar Rauf & Analysis of data and quality insurer. \\
\hline
\end{tabular}

